\title{
Cyclophosphamide, epirubicin and fluorouracil chemotherapy-induced alteration of haemostasis markers in breast cancer patients
}

\author{
ZHI WANG ${ }^{1,2}$, CHENGXUE DANG $^{3}, \mathrm{KUN}_{\mathrm{ZHU}}{ }^{3}$, YONG ZHANG $^{3}$, DONGMIN CHANG $^{3}$, \\ PENG XIA ${ }^{3}$, YONGCHUN SONG ${ }^{3}$ and $\mathrm{KANG} \mathrm{LI}^{3}$ \\ ${ }^{1}$ Xi'an Jiaotong University College of Medicine, Xi'an, Shaanxi 710049; ${ }^{2}$ Tuberculosis Hospital of Shaanxi Province, \\ Xi'an, Shaanxi 710100; ${ }^{3}$ Department of Oncology Surgery, First Affiliated Hospital of Xi'an Jiaotong University, \\ Xi'an, Shaanxi 710061, P.R. China
}

Received October 30, 2014; Accepted January 8, 2015

DOI: $10.3892 / \mathrm{mco} .2015 .584$

\begin{abstract}
The occurrence of chemotherapy-induced alterations in markers of haemostasis during chemotherapy has been reported previously. However, the change of the haemostasis markers in the cyclophosphamide, epirubicin and fluorouracil (CEF) regimen remains unclear. The aim of the present study was to identify the change of the haemostasis markers during systemic chemotherapy $\left(600 \mathrm{mg} / \mathrm{m}^{2}\right.$ cyclophosphamide, $80 \mathrm{mg} / \mathrm{m}^{2}$ epirubicin and $500 \mathrm{mg} / \mathrm{m}^{2}$ fluorouracil; four courses over 21 days) to investigate its influence on the haemostasis markers of breast cancer patients and to discuss the requirement of anticoagulation therapy. D-dimer, activated partial thromboplastin time (APTT), prothrombin time (PT) and fibrinogen (FIB) values were obtained before chemotherapy and on days 1, 4, 7 and 21 . The results show that PT, D-dimer and FIB were not prolonged prior to chemotherapy compared to that under the control. APTT was prolonged until day 4 . The levels of D-dimer and APTT were significantly higher compared to those of the breast cancer patients before receiving chemotherapy and controls on days 1, 4, 7 and 21 after chemotherapy. Alteration of the haemostasis markers occurred in the breast cancer patients under the CEF chemotherapy regimen. As there is an increased risk of deep vein thrombosis and pool prognosis of cancer patients, anticoagulant therapy should be considered.
\end{abstract}

Correspondence to: Dr Kang Li, Department of Oncology Surgery, First Affiliated Hospital of Xi'an Jiaotong University, 277 Yanta West Road, Xi'an, Shaanxi 710061, P.R. China

E-mail: 1809648@qq.com

Key words: breast cancer, D-dimer, cyclophosphamide, epirubicin, fluorouracil, chemotherapy, deep vein thrombosis

\section{Introduction}

The incidence of the hypercoagulable state in occult or overt malignancy patients has been observed since the first observation in 1865 by Trousseau. Patients with cancer are at a higher risk of developing venous thromboembolism (VTE), as confirmed in numerous studies. The reason for developing VTE is due to pathology multifactorials, such as the toxic effect on vascular endothelium, and upregulation of platelet and monocyte activity $(1,2)$. Thus far, the mechanisms according to which cancer cells contribute to the hypercoagulable state are complex and not completely understood. In the hypercoagulable state, tumor cells are packaged by a polymerid of hemaleucin and platelets so that they escape from immunological surveillance $(3,4)$. This state directly facilitates the production of thrombin and numerous signal conduction mechanisms are activated to enhance the production of new tumor vessels and to promote metastasis.

As the use of anticoagulants may be associated with an unacceptably high risk of bleeding complications, investigations have focused on discovering a marker to identify patients at the highest risk for VTE and poor prognosis who would benefit from careful prophylaxis (5). Coagulation-related markers, including thrombin-antithrombin, fibrinogen (FIB), D-dimer, tissue factor (TF), platelet count, activated partial thromboplastin time (APTT) and prothrombin time (PT), have been assayed by investigators to evaluate the state of the haemostatic and procoagulant systems. Alteration of these parameters in malignancy patients have been found in certain studies, and specific alterations have been assumed to be associated with malignancy-induced hypercoagulable state $(6,7)$.

Several researchers have reported that chemotherapy is able to induce alterations in markers of coagulation in breast cancer patients (8). However, few have regarded the change of haemostasis markers in specific regimens commonly used in breast cancer patients. The cyclophosphamide, epirubicin and fluorouracil (CEF) regimen as an adjuvant therapy for breast cancer has widespread implementation in China. The toxic effects, such as granulocytopenia, nausea and vomiting, have been widely investigated and have already 
satisfied the therapeutic effect. Recently, certain studies and clinical activities have begun to focus on the measurement of coagulation markers and anticoagulant treatment $(9,10)$. The proper control of the hypercoagulabale state may be a novel breakthrough for an improved therapeutic effect and lower risk. The present study aimed to investigate the effect of the chemotherapy-induced change in haemostasis biomarkers of the CEF regimen and to assess the risk of deep vein thrombosis and other short- and long-term complications. According to the results and previous studies, the addition of anticoagulant treatment will be discussed to improve the therapeutic effect for chemotherapy.

\section{Patients and methods}

Patients. A total of 80 female patients [median age, 50 years (range, 34-72 years)] commencing chemotherapy for breast cancer in the Department of Oncology Surgery, First Affiliated Hospital of Xi'an Jiaotong University (Shaanxi, China) and 80 female controls [median age, 52 years (range, 32-76 years)] with no history of cancer were recruited who met the following criteria: Female, Karnofsky performance score $>80$, no history of hematopoietic system disease, and laboratory examination confirmed normal heart, liver and kidney function. Exclusion criteria were heart failure as defined by the New York Heart Association (11), hemodynamic instability, hepatic dysfunction or renal dysfunction. The study was approved by the Ethics Committee of The First Affiliated Hospital of Xi'an Jiaotong University. All the patients received CEF regimen chemotherapy. Patients with operable breast cancer received cyclophosphamide at $600 \mathrm{mg} / \mathrm{m}^{2}$, epirubicin at $80 \mathrm{mg} / \mathrm{m}^{2}$ and fluorouracil at $500 \mathrm{mg} / \mathrm{m}^{2}$ every 21 days for four cycles.

Of all the patients, 18 patients exhibited state I breast cancer, 22 had state IIa, 35 had state IIb and five exhibited state IIIa (Table I). Infiltrating ductal carcinoma was observed in 61 patients, infiltrating lobular carcinoma in 16 patients, colloid carcinoma in two patients and erebriform carcinoma in one patient (Table II).

Blood sampling and methods. Markers of haemostasis, such as FIB, D-dimer, PT and APTT, were measured before chemotherapy and the state of haemostatic and procoagulant systems following commencement of chemotherapy in all the patients were evaluated at $24 \mathrm{~h}$ on days 4,7 and 21 . Venous blood punctuation sampling was performed at the antecubital fossa. Within $2 \mathrm{~h}$ after being collected into tubes, all the specimens containing anticoagulants were separated as heparin. Serum samples were allowed to clot at room temperature. All the samples were centrifuged for $20 \mathrm{~min}$ at $41^{\circ} \mathrm{C}$ and $2,500 \mathrm{x} \mathrm{g}$ and the plasma or serum was removed from the cells. Serum and plasma samples were subsequently divided into $0.3 \mathrm{ml}$ aliquots.

All the patients were pathologically diagnosed with breast cancer and were administered in The First Affiliated Hospital of Xi'an Jiaotong University. In total, 62 received modified radical mastectomy or breast-conserving surgery on the basis of the tumor size, position, preoperational biopsy results and imaging examination. Colony-stimulating factor and other supportive treatments were administered to ensure the tolerance of patients in the whole treatment. When any presymptom
Table I. Carcinoma states in breast cancer patients receiving chemotherapy.

\begin{tabular}{lc}
\hline Carcinoma states & No. of patients \\
\hline State & \\
I & 18 \\
IIa & 22 \\
IIb & 35 \\
IIIa & 5 \\
\hline
\end{tabular}

Table II. Pathological type in breast cancer patients receiving chemotherapy.

Pathological type No. of patients

Infiltrating ductal carcinoma 61

Infiltrating lobular carcinoma 16

Colloid carcinoma

2

Erebriform carcinoma

of a fatal event was identified during chemotherapy, the treatment was stopped immediately. A prospective cohort study was undertaken based on the above condition. All the patients provided written informed consent. The study was approved by the Conduct of Human Ethics Committee of Xi'an Jiaotong University.

Statistical analysis. Data on PT, APTT, FIB and D-dimer were assayed by standard laboratory methods with a Sysmex CA-7000 (Sysmex Corporation, Kobe, Japan) automated blood coagulation analyzer with the appropriate reagents and standards. Laboratory analyses were performed in a blinded fashion. APTT and PT data were reported as mean (confidence interval) and D-dimer and FIB data were recorded and reported by geometric means (confidence interval). The analysis of the values in patients prior and subsequent to chemotherapy was performed by repeated measure analysis of variance; the analysis of values between patients and controls was conducted by one-way analysis of variance. $\mathrm{P}<0.05$ was considered to indicate a statistically significant difference.

\section{Results}

Changes in the haemostasis markers during systemic chemotherapy. In total, 71 of the 80 patients completed the therapy. Six patients did not complete the treatment due to myelosuppression, including thrombocytopenia and penineutroa. A total of three patients withdrew due to non-hematological toxic responses, such as strong dizziness, nausea, vomiting and declined appetite. The value of D-dimer was significantly different between prechemotherapy and control patients $(\mathrm{P}<0.05)$, while APTT, PT and FIB were not altered in breast cancer patients compared to the controls prior to chemotherapy ( $\mathrm{P}>0.05$ ) (Fig. 1). The value of coagulation parameters 

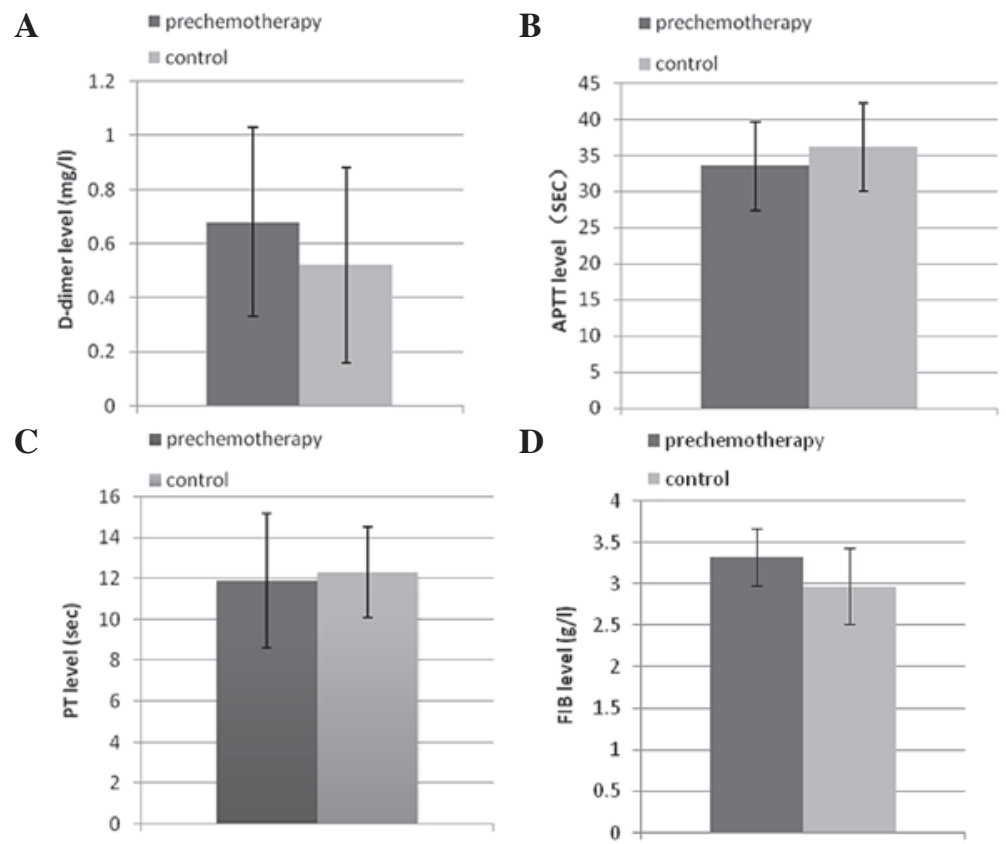

Figure 1. Comparative analysis between prechemotherapy and controls of (A) D-dimer, (B) activated partial thromboplastin time (APTT), (C) prothrombin time (PT) and (D) fibrinogen (FIB). The value of D-dimer was significantly different between prechemotherapy and control (P<0.05), while APTT, PT and FIB were not altered in breast cancer patients compared to the controls prior to chemotherapy $(\mathrm{P}>0.05)$.
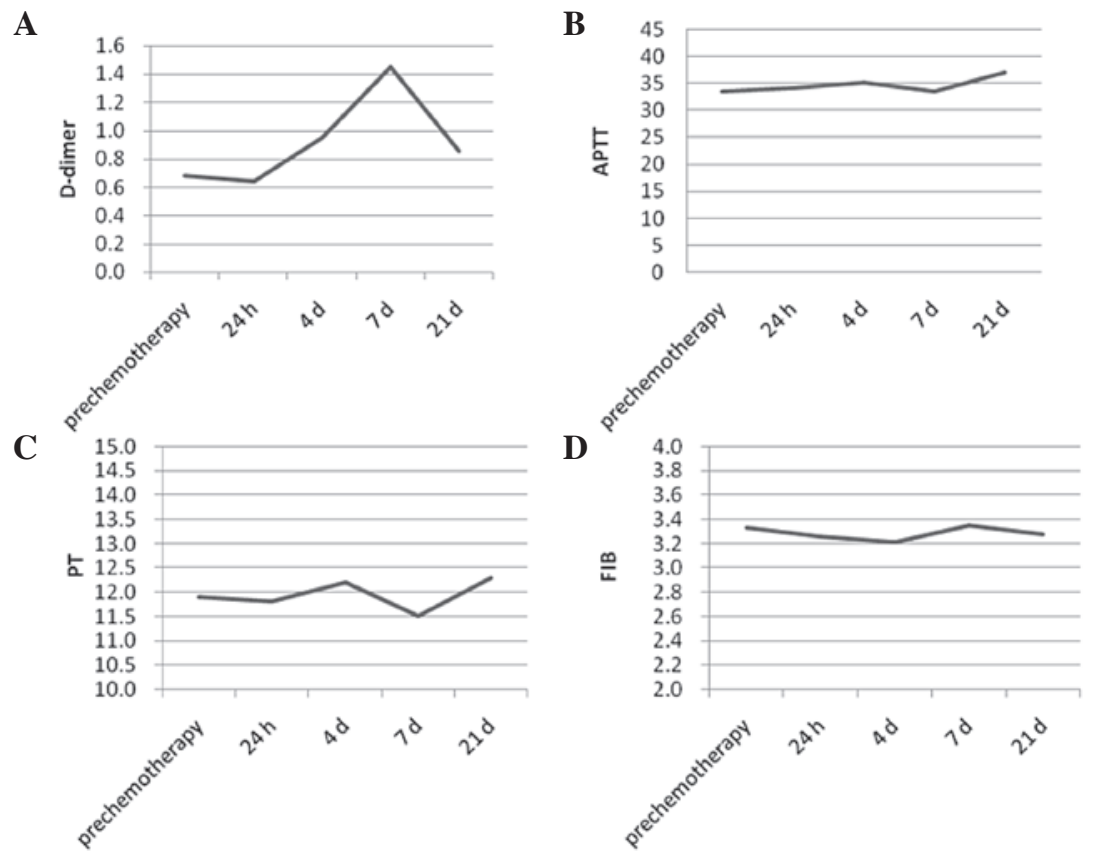

Figure 2. Coagulation parameter values in response to chemotherapy demonstrated that (C) prothrombin time (PT) was not significantly different among days 1, 4, 7 and 21 (repeated-measures analysis) together with (D) fibrinogen (FIB) and (B) activated partial thromboplastin time (APTT) (P>0.05). The value of (A) D-dimer showed a significant difference during chemotherapy $(\mathrm{P}<0.05)$.

in response to chemotherapy demonstrated a different trend; PT was not significantly different among days $1,4,7$ and 21 (repeated-measures analysis). FIB showed a similar trend to PT. APTT on day 1 was prolonged but was not significantly different in comparison to before the chemotherapy. APTT showed an increase within 4 days after the commencement of chemotherapy and returned to the level of prechemotherapy on day 7, but it remained under normal limits and maintained at this level until day 21 ( $\mathrm{P}>0.05)$ (Fig. 2).
The plasma D-dimer level was above the reference value $(1.0 \mathrm{mg} / \mathrm{l})$ in 20 out of the 71 patients with breast cancer prior to chemotherapy $(28.2 \%)$. There was a significant difference between the prechemotherapy group and controls $(\mathrm{P}<0.05)$. Although statistical significance was found in the analysis, 53 out of the 71 values were postoperative, and were not able to exclude the effect of surgery. At $24 \mathrm{~h}$, no significant difference was identified when comparing to the D-dimer level prior to chemotherapy and in patients commencing 
chemotherapy. A marked increase was observed within 4 days after the commencement of chemotherapy and this was more pronounced on day $7(\mathrm{P}<0.001)$. Although the increase in D-dimer was maintained until day 21, the geometric means on day 21 was declining in comparison to those on days 4 and 7 (Table II).

\section{Discussion}

Malignancy is closely associated with the hypercoagulable state. Idiopathic or scandium VTE occurs with the prevalence of $\sim 10 \%$ in malignancy patients as a paraneoplastic phenomenon (6). Necroscopy shows that $50 \%$ of patients with malignancy have thrombogenesis in their body circulation (7). Certain researchers have found that the risk of developing cancer one year after thrombogenesis is 4.4 times that of those without thrombogenesis (12). Tumor itself induces the hypercoagulability state, and the net haemostatic disorder is a characteristic of patients with solid tumors (13).

The aggravation of the hypercoagulability state by chemotherapy has been observed in numerous studies. Several results suggest that the pathogenesis of thrombotic complications may be an important event of the coagulation-fibrinolysis system disorder during chemotherapy (14). The change in the haemostatic and procoagulant systems is reflected by certain TFs and laboratory test values, such as APTT, PT, D-dimer, FIB, cancer procoagulant and plasma vascular endothelial growth factor, and the thromboplastin activation test. A previous study showed a significantly prolonged APTT in low-grade malignant non-Hodgkin's lymphoma (15). APTT, PT, D-dimer and FIB are four widely used measurements that evaluate the coagulation system in China. Therefore, these four measurements were used to observe and analyze the results in the present study.

In numerous studies, D-dimer was observed to be more sensitive and significant in breast cancer and other malignant diseases than other measurements, and it is associated with the complication and prognosis of cancer $(16,17,18)$. D-dimer is a degradation product of fibrin and reflects the fibrin concentration. The development of deep venous thrombosis has been proved to be associated with D-dimer. Increased D-dimer levels have been reported to show the potential presence of silent VTE prior to treatment in ovarian cancer (19). Furthermore, in the majority of relapse or resistance to chemotherapy cases, a further increase of plasma concentration of D-dimer is commonly observed.

The elevated plasma D-dimer values at presentation are associated with a poor overall survival rate, event-free survival and underlying cancer (20). A high preoperative plasma D-dimer level is a marker of advanced tumor stage and short survival rate following curative resection in patients with colorectal cancer (21), as the recovery of the haemostatic balance with effective chemotherapy is associated with the prognosis for patients with gynecological malignancies (22). According to these studies, the importance of D-dimer levels is particularly emphasized in the present study.

For the evaluative role in the curative effect of chemotherapy, the change of D-dimer levels during chemotherapy has been focused on by numerous investigators. Studies have shown that elevated D-dimer plasma levels in patients with lung cancer are associated with a decreased survival rate and a poor response to treatment (16). The result of this study suggests that an imbalance of the coagulation-fibrinolysis system may be a contributing factor in the pathogenesis of thrombotic complications during chemotherapy. D-dimer was also used as a prognosis marker in breast cancer patients (23). Certain results have indicated the presence of a hypercoagulability state in females with operable hormone receptor-negative breast cancer. A significantly high D-dimer level in patients contributed to a poor prognosis, which may be an encouraging marker for the prognosis of operable hormone receptor-negative breast cancer females (24).

The chemotherapy-provoked hypercoagulability state in breast cancer is demonstrated by D-dimer levels in the study of Rella et al (9). Cyclophosphamide, methotrexate and fluoreuracil provokes a trend towards hypercoagulability, which should be considered when chemotherapy is employed in advanced cancer patients with a high risk for thrombosis, or in patients with other risk factors. The present study provided additional clinical data for the field.

The present study showed that coagulation-fibrinolysis manifested a hypercoagulability state through D-dimer and APTT during the CEF regiment chemotherapy. The change of D-dimer was more significant compared to APTT and varied during the chemotherapy treatment. D-dimer levels were elevated on day 4 and reached a peak on day 7. The levels reduced on day 21 but remained higher than that in the prechemotherapy and controls. According to the results and other previous studies, day 7 may be of the highest risk for thrombosis. The significant elevation of D-dimer in patients during chemotherapy may have a poorer prognosis.

Certain studies hypothesized that the D-dimer value could be used in predicting the outcome of cancer. Data showed that the preoperative D-dimer plasma level may not be a predictor of clinical outcome in advanced ovarian cancer patients, while other studies suggested standardizing assays $(25,26)$. However, the positive views were dominating and supported in the present study.

In the present study, the hypothesis was that the proper control of the hypercoagulability state can reduce the risk and improve the prognosis of breast cancer patients receiving chemotherapy. The most sensitive measurement for this could be D-dimer. Certain treatments have been administrated to the hypercoagulability state. There have been studies regarding treatment of the chemotherapy-induced hypercoagulability state $(27,28)$. Improving the prevention and treatment of VTE in cancer patients is required. The American Society of Clinical Oncology and the National Comprehensive Cancer Network has published the guidelines that encourage improving the appropriate use of low molecular weight heparin (LMWH) and other agents to enhance the clinical outcomes in medical cancer patients at risk for VTE and its complications (27). Another study has certified the safety of long-term anticoagulation with LMWH and is effective in reducing the recurrence of VTE in cancer. The role of thromboprophylaxis in ambulatory cancer patients receiving chemotherapy is an area of new insight (28). LMWH was confirmed to be a more effective and safer commonly-used anticoagulant agent (28). However, no prospective clinical studies have addressed the alternation of D-dimer during 
anticoagulant administration chemotherapy. Forthcoming studies should focus on this topic. Although the clinical trials are limited, the beneficial effect of anticoagulant treatment on the survival rate of cancer patients is positively confirmed. Regarding the safety consideration of the anticoagulant therapy, more clinical trials on the suitable dose and the duration of treatment are required in this area. However, for an improved response to treatment and prognosis, the use of anticoagulant therapy is recommended in the present study.

In conclusion, during the CEF regiment chemotherapy, the change of D-dimer was more significant than APTT and was not at the same level during the chemotherapy. D-dimer levels were elevated on day 4 and reached a peak on day 7 . According to the results and other previous studies, day 7 may be of the highest risk for thrombosis. A hypothesis was introduced that the proper control of the hypercoagulability state can reduce the risk and improve the prognosis of breast cancer patients receiving chemotherapy. The most sensitive measurement for this could be D-dimer. For an improved response to treatment and prognosis, the use of anticoagulant therapy is recommended. Regarding the safety consideration of the anticoagulant therapy, more clinical trials on the suitable dose and the duration of treatment are required in this area.

\section{References}

1. Zecchina G, Ghio P, Bosio S, Cravino M, Camaschella C and Scagliotti GV: Reactive thrombocytosis might contribute to chemotherapy-related thrombophilia in patients with lung cancer. Clin Lung Cancer 8: 264-267, 2007.

2. Albertsson P, Lennernas B and Norrby K: Low-dose continuous 5-fluorouracil infusion stimulates VEGF-A-mediated angiogenesis. Acta Oncol 48: 418-425, 2009.

3. Milanini-Mongiat J, Pouyssegur J and Pages G: Identification of two Sp1 phosphorylation sites for $\mathrm{p} 42 / \mathrm{p} 44$ mitogen-activated protein kinases: their implication in vascular endothelial growth factor gene transcription. J Biol Chem 277: 20631-20639, 2002.

4. Karpatkin S: Does hypercoagulability awaken dormant tumor cells in the host? J Thromb Haemost 2: 2103-2106, 2004.

5. Sood SL: Cancer-associated thrombosis. Curr Opin Hematol 16 : 378-385, 2009.

6. Rak J, Yu JL, Luyendyk J and Mackman N: Oncogenes, trousseau syndrome, and cancer-related changes in the coagulome of mice and humans. Cancer Res 66: 10643-10646, 2006.

7. Sutherland DE, Weitz IC and Liebman HA: Thromboembolic complications of cancer: epidemiology, pathogenesis, diagnosis, and treatment. Am J Hematol 72: 43-52, 2003.

8. Oberhoff $\mathrm{C}$, Winkler UH, Hoffmann $\mathrm{O}$ and Schindler AE: Adjuvant CMF-chemotherapy and haemostasis. Effect of 'classical' and 'modified' adjuvant CMF-chemotherapy on blood coagulation fibrinolysis in patients with breast cancer. Eur J Gynaecol Oncol 21: 147-152, 2000.

9. Rella C, Coviello M, Giotta F, et al: A prothrombotic state in breast cancer patients treated with adjuvant chemotherapy. Breast Cancer Res Treat 40: 151-159, 1996.

10. Nomura H, Wada H, Mizuno T, et al: Elevated fibrin-related markers in patients with malignant diseases suspected of having thrombotic disorders. Clin Appl Thromb Hemost 16: 266-272, 2010 .
11. American Heart Association: Classes of heart failure. http://www.heart.org/HEARTORG/Conditions/HeartFailure/About Heart Failure/Classes-of-Heat-Failure_UCM 306328 Article. jsp. Accessed September 6, 2011.

12. Baron JA, Gridley G, Weiderpass E, Nyren O and Linet M: Venous thromboembolism and cancer. Lancet 351: 1077-1080, 1998.

13. Garcia-Avello A, Galindo-Alvarez J, Martinez-Molina E, Cesar-Perez J and Navarro JL: Coagulative system activation and fibrinolytic system inhibition activities arise from tumoral draining vein in colon carcinoma. Thromb Res 104: 421-425, 2001.

14. Gabazza EC, Taguchi O, Yamakami T, et al: Alteration of coagulation and fibrinolysis systems after multidrug anticancer therapy for lung cancer. Eur J Cancer 30A: 1276-1281, 1994.

15. Siemens HJ, Gerke P, Steinhoff J, Roth-Isigkeit A, Wagner K and Bruckner S: A prolonged APTT in a patient with a low grade malignant NHL - a case report. Haematologica 87: ELT08, 2002.

16. Tamizifar B, Oghab P and Esfahani MA: The prediction role of D-dimer in recurrence of venous thromboembolism 1-year after anticoagulation discontinuing following idiopathic deep vein thrombosis. J Res Med Sci 19: 586-591, 2014.

17. Arpaia G, Carpenedo M, Verga M, et al: D-dimer before chemotherapy might predict venous thromboembolism. Blood Coagul Fibrinolysis 20: 170-175, 2009.

18. Gadducci A, Marrai R, Baicchi U, et al: Preoperative D-dimer plasma assay is not a predictor of clinical outcome for patients with advanced ovarian cancer. Gynecol Oncol 66: 85-88, 1997.

19. Satoh T, Oki A, Uno K, et al: High incidence of silent venous thromboembolism before treatment in ovarian cancer. $\mathrm{Br} \mathrm{J}$ Cancer 97: 1053-1057, 2007.

20. Lippi G, Franchini M, Biasiutti C, Dellagiacoma G, Salvagno GL and Guidi GC: Increased D-dimer value and occult cancer in the absence of detectable thrombosis. Haematologica 92: e53-e 55, 2007.

21. Oya M, Akiyama Y, Yanagida T, Akao S and Ishikawa H: Plasma D-dimer level in patients with colorectal cancer: its role as a tumor marker. Surg Today 28: 373-378, 1998.

22. Sawaguchi K, Yabushita H, Higuchi K, et al: Effect of remission induction chemotherapy on blood coagulability in patients with gynecological malignancies. Nippon Gan Chiryo Gakkai Shi 24: 798-808, 1989 (In Japanese).

23. Yigit E, Gonullu G, Yucel I, Turgut M, Erdem D and Cakar B: Relation between hemostatic parameters and prognostic/predictive factors in breast cancer. Eur J Intern Med 19: 602-607, 2008

24. Batschauer AP, Figueiredo CP, Bueno EC, et al: D-dimer as a possible prognostic marker of operable hormone receptor-negative breast cancer. Ann Oncol 21: 1267-1272, 2010.

25. Lyman GH: Thromboprophylaxis with low-molecular-weight heparin in medical patients with cancer. Cancer 115: 5637-5650, 2009.

26. Di Nisio M, Klerk CP, Meijers JC and Büller HR: The prognostic value of the $\mathrm{D}$-dimer test in cancer patients treated with and without low-molecular-weight heparin. J Thromb Haemost 3: 1531-1533, 2005.

27. Sousou T and Khorana AA: New insights into cancer-associated thrombosis. Arterioscler Thromb Vasc Biol 29: 316-320, 2009.

28. Gerotziafas GT, Papageorgiou C, Hatmi M, Samama MM and Elalamy I: Clinical studies with anticoagulants to improve survival in cancer patients. Pathophysiol Haemost Thromb 36: 204-211, 2008. 